\title{
Reprodutibilidade Interobservador da Classificação da Distopia Genital Proposta pela Sociedade Internacional de Continência
}

\author{
Interobserver Reproducibility of Pelvic Organ Prolapse Classification Suggested \\ by the International Continence Society \\ Paulo Cezar Feldner Jr, Leonardo Robson Pinheiro Sobreira Bezerra \\ Emerson Oliveira, Marair Gracio Ferreira Sartori, Edmund Chada Baracat \\ Geraldo Rodrigues de Lima, Manoel João Bastista Castello Girão
}

\begin{abstract}
RESUMO
Objetivo: testar a reprodutibilidade entre observadores das medidas e do estádio da distopia genital pela classificação do prolapso pélvico feminino preconizada pela Sociedade Internacional de Continência (ICS).

Métodos: foram avaliadas 51 pacientes atendidas no setor de Uroginecologia e Cirurgia Vaginal do Departamento de Ginecologia da UNIFESP/EPM durante investigação uroginecológica. Descrevemos a localização dos pontos propostos pela classificação da ICS, sendo dois na parede vaginal anterior, dois no ápice vaginal, dois na parede vaginal posterior, além do hiato genital, corpo perineal e comprimento vaginal total. A seguir, realizamos o estadiamento da distopia genital baseada nesta classificação. O procedimento foi realizado por dois investigadores diferentes sem contato prévio entre eles. A reprodutibilidade das nove medidas sitio-específicas e do estádio final foi analisada pela correlação de Pearson e a média dos pontos específicos pelo teste de t-pareado.

Resultados: houve correlação significativa e substancial para as medidas avaliadas. O indice de correlação para o ponto Aa foi de 0,89 (p<0,0001), ponto Ba de 0,90 (p<0,0001), ponto C de 0,97 ( $p<0,0001)$, ponto Ap de 0,72 ( $p<0,0001)$, ponto Bp de 0,84 ( $p<0,0001)$, ponto D de 0,91 $(p<0,0001)$, hiato genital de 0,65 ( $p<0,0001)$, corpo perineal de 0,66 ( $p<0,0001)$ e comprimento vaginal total de 0,73 ( $p<0,0001)$. Também não se observou variação na média das medidas realizadas pelos dois examinadores. Da mesma forma, o estádio final da distopia foi altamente reprodutivel ( $r=0,81, p<0,0001)$. Em nenhuma paciente houve variação maior que um estádio, sendo idênticos em $86,2 \%$ dos casos.

Conclusões: existe reprodutibilidade nas medidas obtidas pelo sistema de classificação da distopia genital da Sociedade Internacional de Continência.
\end{abstract}

PALAVRAS-CHAVE: Prolapso genital. Distopia. Incontinência urinária.

\section{Introdução}

A avaliação da anatomia do assoalho pélvico e das distopias genitais tem sido grande desafio para a especialidade. Embora a disfunção

Universidade Federal de São Paulo - EPM / UNIFESP; Departamento de Ginecologia da Escola Paulista de Medicina, Setor de Uroginecologia e Cirurgia Vaginal

Correspondência:

Paulo Cezar Feldner Jr

Rua Mirassol, 272, apto 61 - Vila Clementino

04044-010 - São Paulo - SP

e-mail: pfeldner@alfa.epm.br do assoalho pélvico seja muito freqüente, seu estudo encontrava dificuldades pela ausência de sistema universalmente aceito para descrição da posição anatômica dos órgãos pélvicos. Muitos relatos usavam termos indefinidos. Essa falta de padronização não permitia comparações de estudos publicados entre diferentes instituições e, tampouco, avaliações longitudinais ${ }^{1}$.

Os diversos modos de registro dos achados do exame físico impediam a difusão dos estudos e a comparação entre os dados ao longo do tempo. Os relatos que descreviam os resultados dos procedimentos cirúrgicos para o tratamento da 
distopia genital também não encontravam padrões de evidência científica para análise ${ }^{1}$.

Em 1995, o Comitê Internacional Multidisciplinar composto por membros da ICS (International Continence Society), da Sociedade Americana de Uroginecologia e da Sociedade dos Cirurgiões Ginecologistas elaborou protocolo de padronização ${ }^{2}$. Durante os anos subseqüentes, pequenas revisões foram feitas e estudos de reprodutibilidade em centros nos EUA e Europa foram realizados ${ }^{3,4}$. Documentou-se a capacidade de realização do método, a variabilidade inter e intra-observador e, sobretudo, a utilidade clínica do sistema. Em 1996, o documento foi adotado e validado formalmente pela ICS, pela Sociedade Americana de Uroginecologia e pela Sociedade dos Cirurgiões Ginecologistas ${ }^{5}$.

Neste sistema de estadiamento, os pontos de medida são estabelecidos com o intuito de aferir valores numéricos à intensidade do prolapso, não se baseando na gravidade dos sintomas clínicos ou em outros achados. Trata-se de sistema descritivo que contém vários componentes a serem avaliados. Tem como objetivo padronizar a terminologia e a classificação da disfunção do assoalho pélvico. Sua reprodutibilidade e utilidade clínica já foram testadas, sendo que esta padronização vem sendo utilizada de forma rotineira em publicações internacionais ${ }^{5,6}$.

O objetivo deste estudo foi avaliar, em nosso meio, a reprodutibilidade entre dois diferentes observadores das medidas e do estádio da distopia genital propostos por este sistema de classificação.

\section{Métodos}

Foi realizado estudo transversal no qual foram incluídas 51 mulheres atendidas no periodo de junho a setembro de 2002, no setor de Uroginecologia e Cirurgia Vaginal da UNIFESP/ EPM.

Todas as pacientes foram avaliadas por anamnese e exame físico. A anamnese consistiu de questionário-padrão pelo qual se inquiriu sobre queixas relativas à distopia genital. Também inquirimos outros sintomas uroginecológicos como a perda de urina aos esforços, urgência miccional, urge-incontinência, disúria, hematúria, polaciúria, repleção vesical, necessidade de força para iniciar a diurese, esvaziamento incompleto da bexiga, enurese noturna e perda constante de urina.

As pacientes foram examinadas por dois investigadores diferentes, sem que um tivesse conhecimento dos resultados obtidos pelo outro. O exame foi realizado pela equipe de pósgraduandos do setor, sendo que os examinadores tinham experiência variável em uroginecologia. Registraram-se os dados em folha padronizada para a coleta dos achados clínicos. As pacientes foram avaliadas em posição ginecológica, tendo a mesa ginecológica inclinação de $45^{\circ}$. Pediu-se que realizassem manobra de Valsalva para se visualizar a máxima protrusão ou prolapso.

Segundo orientação da padronização da terminologia proposta pela ICS ${ }^{5}$, evitou-se o uso de termos como cistocele, retocele, enterocele ou junção uretrovesical. Portanto, para o propósito da discussão dessa nova terminologia, o prolapso pélvico feminino incluiu o prolapso de parede vaginal anterior (anteriormente conhecido como cistocele), o prolapso da cúpula vaginal ou uterino e o prolapso de parede vaginal posterior (anteriormente conhecido como retocele e enterocele).

A descrição dos pontos propostos pela classificação pode ser resumida da seguinte forma: seis pontos na vagina (dois na parede anterior, dois no ápice vaginal e dois na parede posterior), sempre relacionados com o plano do hímen, este definido como ponto de referência.

As posições dos pontos são dadas em centímetros com números negativos quando acima do hímen. Como exemplo, o colo que se encontra em situação intravaginal, $3 \mathrm{~cm}$ proximal ao hímen, terá sua posição indicada como a $-3 \mathrm{~cm}$. As posições são expressas em números positivos quando distalmente ao hímen. Como exemplo, o colo que se encontra prolapsado $3 \mathrm{~cm}$ ao hímen terá sua posição indicada como $a+3 \mathrm{~cm}$ ).

Os seis pontos anatômicos citados são: 1 : ponto Aa - localizado na linha média da parede vaginal anterior, $3 \mathrm{~cm}$ proximal ao meato externo da uretra; 2: ponto $\mathrm{Ba}$ - caracterizado como a posição mais distal de qualquer parte da parede vaginal anterior, correspondendo ao ápice do prolapso da parede vaginal anterior; 3 : ponto $\mathrm{C}$ correspondente à parte mais distal da cérvix uterina ou da cúpula vaginal se a paciente houvesse sido submetida a histerectomia; 4: ponto $D$ - reflete a localização do fórnice posterior na mulher que ainda tem colo uterino. Representa a altura em que os ligamentos útero-sacrais se ligam à cérvix uterina. $\mathrm{O}$ ponto $\mathrm{D}$ é omitido na ausência do colo uterino; 5: ponto Ap - localiza-se na linha média da parede vaginal posterior, $3 \mathrm{~cm}$ proximal à carúncula himenal; 6: ponto $\mathrm{Bp}$ - representa a posição mais distal de qualquer parte da parede vaginal posterior, correspondendo ao ápice do prolapso desta parede.

As próximas três medidas completam a descrição da distopia genital: 1: hiato genital 
(HG) - medido do ponto médio do meato externo da uretra à linha média posterior na altura da carúncula himenal; 2: corpo perineal (CP) medido da margem posterior do hiato genital ao ponto médio do orificio anal; 3: comprimento vaginal total (CVT) - é a distância desde carúncula himenal até o ponto mais profundo da vagina quando os pontos $\mathrm{C}$ ou $\mathrm{D}$ estão em suas posições normais. Os diversos pontos de medida estão mostrados na Figura 1, reproduzida de Bump et al. ${ }^{5}$.

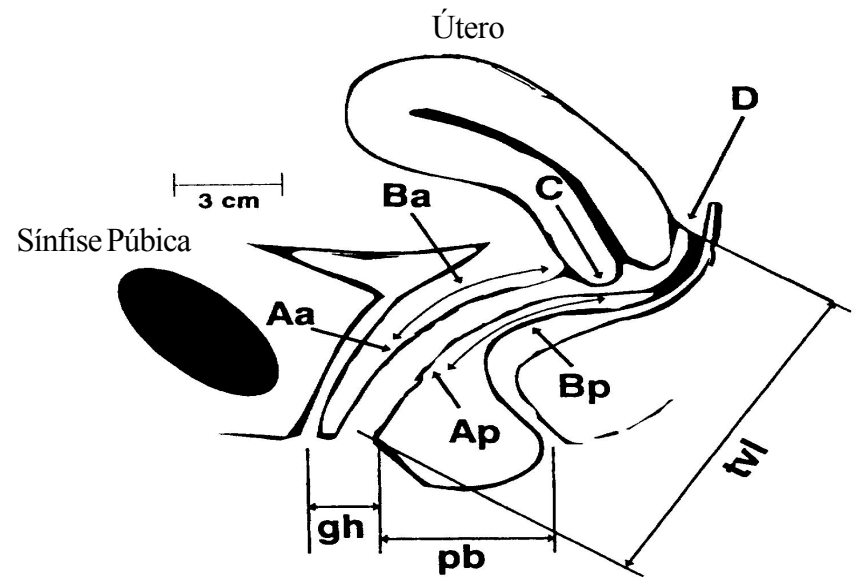

Figura 1 - Padronização dos pontos de medida pelo sistema de classificação da distopia genital preconizado pela Sociedade Internacional de Continência (Bump etal. ${ }^{5}$ ).

Aa: Ponto Aa

$\mathrm{Ba}$ : Ponto $\mathrm{Ba}$

C: Ponto C

D:Ponto D

Ap: Ponto Ap

Bp: Ponto Bp

gh: Hiato genital (HG)

$\mathrm{pb}$ : Corpo perineal (CP)

tvl: Comprimento vaginal total (CVT)

A coleta dos dados foi assim padronizada: a primeira medida obtida, antes de qualquer manipulação, foi do hiato genital e do corpo perineal com o histerômetro ou a régua plástica, sob esforço da paciente. Se o prolapso ultrapassasse o anel himenal, as medidas dos pontos externos seriam obtidas primeiramente. Se não houvesse prolapso, as medidas dos pontos A e $B$ posteriores e anteriores seriam realizadas com espéculo reduzindo a parede vaginal oposta. O espéculo vaginal foi colocado e lentamente retirado com a paciente realizando manobra de Valsalva. Mediram-se, então, os pontos C e D com o histerômetro. O comprimento vaginal total foi medido pelo reposicionamento digital do ápice vaginal em sua posição anatômica, medindo-se a profundidade com o histerômetro, com a paciente em repouso.
Uma vez que as nove medidas foram colhidas, agruparam-se as pacientes de acordo com um dos cinco estádios do prolapso pélvico, que incluem estágio 0: não há prolapso, com os pontos $\mathrm{Aa}, \mathrm{Ba}, \mathrm{Ap}, \mathrm{Bp}$ a $-3 \mathrm{~cm}$ e os pontos $\mathrm{C}$ ou $\mathrm{D}$ dentro do intervalo CVT e CVT $-2 \mathrm{~cm}$; estágio I: não se encontram os critérios para o estágio 0 , mas o ponto de referência mais distal do prolapso é maior que $1 \mathrm{~cm}$ acima do hímen; estágio II: o ponto de referência mais distal do prolapso está entre $-1 \mathrm{e}+1 \mathrm{~cm}$ do plano do hímen; estágio III: a porção mais distal do prolapso está além de 1 $\mathrm{cm}$ distal ao plano do hímen, mas ultrapassa a distância do CVT -2 cm; estágio IV: em essência, seria completa eversão. Na maioria dos casos o ponto mais externo seria a cérvix ou o fundo de saco vaginal.

De maneira geral, a ausência de prolapso em qualquer compartimento foi classificada como estágio 0 . O prolapso genital foi definido como o encontro, ao exame físico, de procidência que atingisse ou ultrapassasse o estágio I. A classificação é feita com base no compartimento que mais se exterioriza.

Todos os métodos, definições e termos utilizados seguiram a padronização da Sociedade Internacional de Continência ${ }^{7}$.

O estudo foi desenhado para se detectarem alterações absolutas de $20 \%$ ou mais entre os grupos. Considerando-se que existem cinco estádios, a diferença de um estádio entre os examinadores poderia representar tal alteração. Para assegurar poder de força do teste estatístico de pelo menos $80 \%$ e limitar a chance de erro tipo I a $5 \%(\alpha<5 \%)$, o número de pacientes necessário seria de no mínimo 50 .

Para averiguar a associação entre as nove medidas sítio-específicas e do estádio da distopia genital, obtidas pelos dois diferentes observadores, utilizamos o coeficiente de correlação de Pearson. Este é teste paramétrico para verificar quão linearmente as variáveis, medidas em escala contínua e com distribuição normal, estão relacionadas ${ }^{8}$. Coeficientes entre 0,81-1,00 foram considerados quase perfeitos, aqueles entre $0,61-0,80$ são substanciais e aqueles entre 0,41-0,60 foram considerados como moderados. A diferença entre a média das mensurações realizadas pelos dois diferentes examinadores foi analisada pelo teste de t-pareado. A análise estatística foi feita utilizando o software Analyse-it Ltd., versão 1.64.

O projeto foi aprovado pelo Comitê de Ética em Pesquisa da Universidade Federal de São Paulo - Escola Paulista de Medicina (UNIFESP-EPM). 
Resultados

Foram avaliadas 51 pacientes com queixas clínicas de distopia genital e/ou de perda urinária. A média de idade foi de 51,2 anos (com variação entre 19 e 82), sendo que 24 (47\%) encontravamse no menacme e 27 (53\%) na pós-menopausa. A paridade média foi de 3,9 filhos (0-18).

A comparação interobservador entre os estádios do prolapso genital pode ser observada na Tabela 1. Não houve variação maior que um estádio. Houve coincidência quanto à classificação em 44 das 51 pacientes avaliadas $(86,2 \%)$. Observamos que o maior grau de correlação ocorreu quando as pacientes encontravam-se no estádio III.

Tabela 1 - Comparação interobservador dos estádios do prolapso genital.

\begin{tabular}{lccccc}
\hline Obs. 1 & O & I & II & III & IV \\
\hline O & - & - & - & - & - \\
I & - & 5 & 2 & - & - \\
II & - & 4 & 27 & 1 & - \\
III & - & - & - & 12 & - \\
IV & - & - & - & - & -
\end{tabular}

Obs. 1: Examinador 1 e Obs. 2: Examinador 2

Estadio 0: 0/0

Estadio : $5 / 7$ (71,4\% de coincidência entre os observadores)

Estadio ll: $27 / 32$ (84,3\% de coincidência entre os observadores)

Estadio III: 12/12 (100\% de coincidência entre os observadores)

Estadio IV: $0 / 0$

A Tabela 2 demonstra que a correlação entre as medidas obtidas pelos dois diferentes examinadores para cada um dos pontos específicos e para o estadiamento do prolapso pélvico segundo a padronização adotada pela ICS foi altamente substancial e significante quando avaliada pelo coeficiente de Pearson. Observamos que a determinação da localização do ponto $\mathrm{C}$ foi a com maior correlação $(r=0,97, p<0,001)$.

Os valores médios de cada um dos nove pontos de medida desta nova classificação, de acordo com cada examinador, estão representados na Tabela 3. Não encontramos diferenças significativas entre a média, em centímetros, da localização de cada um dos pontos específicos obtidos pelos dois diferentes examinadores quando avaliamos as medidas pelo teste de $t$ pareado ( $\mathrm{p}$ não significativo para todas as medidas). Observamos que a média da localização do ponto D obtida pelos dois examinadores foi praticamente idêntica. Da mesma forma, as diferenças entre todas as demais medidas foram clinicamente insignificantes, com variação de no máximo $2 \mathrm{~cm}$ entre os examinadores.
Tabela 2 - Índice de correlação entre as medidas obtidas pelos dois diferentes examinadores para cada um dos pontos específicos e para o estádio na padronização do prolapso pélvico adotada pela ICS.

\begin{tabular}{lcc}
\hline & $\begin{array}{c}\text { Índice de correlação } \\
\text { de Pearson (IC 95\%) }\end{array}$ & valor de p \\
\hline Estádio & $0,81(0,69-0,89)$ & $<0,0001$ \\
Ponto Aa & $0,89(0,81-0,94)$ & $<0,0001$ \\
Ponto Ba & $0,90(0,82-0,94)$ & $<0,0001$ \\
Ponto C & $0,97(0,94-0,98)$ & $<0,0001$ \\
Ponto Ap & $0,72(0,55-0,83)$ & $<0,0001$ \\
Ponto Bp & $0,84(0,74-0,91)$ & $<0,0001$ \\
Ponto D & $0,91(0,85-0,95)$ & $<0,0001$ \\
Hiato genital & $0,65(0,45-0,78)$ & $<0,0001$ \\
Corpo perineal & $0,66(0,47-0,79)$ & $<0,0001$ \\
Comprimento vaginal total & $0,73(0,57-0,84)$ & $<0,0001$
\end{tabular}

IC: Intervalo de confiança. Localização dos pontos (Figura 1).

Tabela 3 - Comparação entre a média ( \pm desvio padrão), em centímetros, da localização de cada um dos pontos específicos obtidos pelos dois diferentes examinadores pelo teste t-pareado.

\begin{tabular}{|c|c|c|c|}
\hline & $\begin{array}{c}\text { Examinador } 1 \\
\text { Média + DP }\end{array}$ & $\begin{array}{l}\text { Examinador } 2 \\
\text { Média + DP }\end{array}$ & $p$ \\
\hline Ponto $\mathrm{Aa}$ & $-0,5 \pm 1,64$ & $-0,7 \pm 1,66$ & 0,07 \\
\hline Ponto $\mathrm{Ba}$ & $-0,3 \pm 1,96$ & $-0,1 \pm 2,08$ & 0,55 \\
\hline Ponto C & $-3,8 \pm 4,12$ & $-3,9 \pm 4,21$ & 0,35 \\
\hline Ponto Ap & $-1,6 \pm 0,96$ & $-1,6 \pm 0,91$ & 0,84 \\
\hline Ponto Bp & $-1,2 \pm 0,26$ & $-1,1 \pm 0,28$ & 0,43 \\
\hline Ponto D & $-5,9 \pm 4,33$ & $-5,9 \pm 4,02$ & 1,00 \\
\hline Hiato genital & $3,4 \pm 1,04$ & $3,5 \pm 1,18$ & 0,24 \\
\hline Corpo perineal & $3,4 \pm 1,02$ & $3,3 \pm 1,08$ & 0,26 \\
\hline Comprimento vVaginal total & $8,6 \pm 1,37$ & $8,5 \pm 1,44$ & 0,50 \\
\hline
\end{tabular}

DP: Desvio padrão. Localização dos pontos (Figura 1).

\section{Discussão}

Nas últimas décadas, diversos autores vêm tentando classificar o prolapso pélvico valendose de critérios de gravidade progressiva em relação a referenciais fixos. Porges ${ }^{9}$, em 1963, classificou o prolapso genital como leve ou de $1^{\circ}$ grau moderado ou de $2^{\circ}$ grau e grave ou de $3^{\circ}$ grau. Já Baden et al. ${ }^{10}$, em 1968, propuseram o perfil vaginal. Beecham ${ }^{11}$, em 1980, adotou o sistema de graus, valendo-se do ponto de referência no anel himenal.

A classificação de Baden et al. ${ }^{10}$ modificada por Juma et al. ${ }^{12}$, em 1993, é a de uso mais difundido em nosso meio. Utilizam-se denominações distintas para os diferentes compartimentos. A distopia da uretra e da bexiga é classificada nos seguintes graus: $1^{\circ}, 2^{\circ}, 3^{\circ}$ e $4^{\circ}$ graus. A 
distopia da parede vaginal posterior é classificada de acordo com graus de severidade: leve, moderada e acentuada. Já o prolapso uterino é classificado em $1^{\circ}, 2^{\circ}$ e $3^{\circ}$ graus, com o colo pinçado e tracionado durante o exame genital. Contudo, a utilização de termos imprecisos como terço inferior da vagina, além da realização do exame com tração, certamente superestima os achados.

$\mathrm{Na}$ elaboração do protocolo de condutas em 1996, a AHCPR (Agency for Health Care Policy and Research) ${ }^{13}$ concluiu que a literatura cirúrgica sobre o tratamento da disfunção do assoalho pélvico é deficiente. Há limitações na descrição da população estudada, do tipo de incontinência, dos métodos para o diagnóstico, das técnicas utilizadas no procedimento cirúrgico e do resultado das diferentes condutas. Black e Downs ${ }^{14}$ corroboram esses achados ao documentar a baixa qualidade dos resultados do tratamento cirúrgico. Embora essas conclusões tenham sido feitas para incontinência urinária de esforço, avaliação similar também pode ser feita para o diagnóstico e a classificação da distopia genital ${ }^{15}$.

$\mathrm{O} \mathrm{NIH}$ (National Institutes of Health) e o NICHD (National Institute of Children Health and Human Development) estabeleceram padrões após avaliar o estado atual da pesquisa básica, epidemiológica e clínica sobre prolapso pélvico feminino $^{1}$. O obstáculo determinante foi a inexistência de padronização da terminologia. Nenhum dos sistemas de graduação tinha sido adequadamente validado com respeito à sua reprodutibilidade, variabilidade inter e intra-observador, ou ainda sobre o significado clínico dos diferentes graus.

Com o objetivo de preencher esta importante lacuna e padronizar a nomenclatura, a forma de mensuração e a classificação das disfunções do assoalho pélvico, a ICS adotou e validou esta nova padronização ${ }^{2}$.

Kobak et al. ${ }^{4}$, avaliando 49 pacientes, evidenciaram importante reprodutibilidade do método. Todos os pontos anatômicos tiveram correlação significativa e uma alta precisão entre os examinadores foi observada. Da mesma forma, o estadiamento da distopia genital teve elevada concordância entre os observadores, com o coeficiente de correlação de 0,79 ( $\mathrm{p}<0,001)$.

Hall et al. ${ }^{6}$ demonstraram significativa reprodutibilidade inter e intra-observador da padronização em 48 mulheres avaliadas. O estadiamento e subestadiamento foram altamente reprodutiveis, não havendo variação maior que um estádio. Em 69 e 64\% dos casos inter e intraobservador, respectivamente, os estádios foram coincidentes. Observaram também que a posição em pé das pacientes determinou maior acentuação da distopia genital. O tempo médio gasto foi de 2,0 minutos para examinador experiente e 3,7 minutos para novo examinador. Os pesquisadores concluíram que houve boa reprodutibilidade de medidas independente da experiência do examinador.

Em contrapartida, Bland et al. ${ }^{16}$ utilizaram a padronização da ICS e demonstraram mudanças na classificação em mulheres na perimenopausa no período de 1 ano. Os autores evidenciaram diminuição significativa com relação ao ponto $\mathrm{C}$, ponto $\mathrm{D}$ e comprimento vaginal total. Contudo, tal diferença esteve relacionada com o aumento da distopia genital do segmento apical da vagina no intervalo de tempo avaliado.

Nossos dados, em concordância com a literatura, demonstraram substancial reprodutibilidade interobservador tanto na quantificação das medidas dos pontos específicos quanto na determinação do estádio do prolapso pélvico. Não houve diferenças significativas na mensuração dos diversos pontos entre os diferentes examinadores. Os achados foram coincidentes em $86,2 \%$ dos casos na determinação do estádio da distopia genital. Nos casos de não-concordância, a diferença foi de apenas um estádio, não representando alterações clínicas significativas.

Embora esta padronização não determine qual a melhor posição na qual a paciente deva ser examinada, esta deve ser especificada durante a descrição dos achados, uma vez que a posição em pé parece acentuar a gravidade da distopia ${ }^{6,17,18}$. Barber et al. ${ }^{18}$ observaram aumento significativo na magnitude do prolapso genital em oito dos pontos medidos, com exceção do comprimento vaginal total. De acordo com os autores, $48 \%$ das pacientes tinham pelo menos uma medida aumentada em $2 \mathrm{~cm}$ ou mais quando examinadas na posição de pé. No presente estudo, as pacientes foram examinadas na posição ginecológica.

Steele et al. ${ }^{19}$ evidenciaram que esta padronização é relativamente fácil no ensino e na aprendizagem. Utilizando apresentação em vídeo, demonstraram aumento significativo na habilidade de interpretação dos achados quando expressos nesta nova terminologia.

O sistema de padronização é indiscutivelmente necessário. Essa determinação busca tão somente estabelecer padrão sobre a nomenclatura da disfunção de assoalho pélvico, devendo ser rotineiramente utilizada para as publicações internacionais. Se populações estão sendo descritas e os resultados de várias opções terapêuticas comparados, os dados devem ser registrados utilizando-se a mesma terminologia padronizada. 


\section{ABSTRACT}

Purpose: to determine interobserver reliability of site-specific measurements and stages according to the proposed International Continence Society prolapse terminology document.

Methods: we analyzed 51 women during urogynecological investigation performed at the Urogynecology and Vaginal Surgery Sector of UNIFESP / EPM. We recorded the locations of point-specific measures proposed by the International Continence Society (ICS). They are: two in the anterior vaginal wall, two in the superior vagina, two in the posterior vaginal wall, genital hiatus, perineal body and total vaginal length. Then we recorded the stage of genital prolapse. Women underwent pelvic examinations by two investigators, each blinded to the results of the other's examination. The reproducibility of the nine site-specific measurements and the summary stage were analyzed using Pearson's correlation coefficient and the median measurements were compared by the paired-t test.

Results: there were substantial and highly significant correlations for each of the nine measurements. Correlation coefficient for point Aa was 0.89 ( $p<0.0001)$, point Ba 0.90 $(p<0.0001)$, point $C 0.97(p<0.0001)$, point Ap de 0.72 $(p<0.0001)$, point Bp $0.84(p<0.0001)$, point $D 0.91$ $(p<0.0001)$, genital hiatus $0.65(p<0.0001)$, perineal body $0.66(p<0.0001)$ e total vaginal length $0.73(p<0.0001)$. We also did not note differences between the means of measurements by the two examiners. Staging was highly reproducible $(r=0.81, p<0.0001)$. In no subject did the stage vary by more than one; in $86,2 \%$, stages were identical. Conclusions: there is a good reproducibility of measures using the system proposed by the International Continence Society prolapse terminology document.

KEYWORDS: Genital prolapse. Genital dystopia. Urinary incontinence.

\section{Referências}

1. Weber AM, Abrams P, Brubaker L, et al. The standardization of terminology for researchers in female pelvic floor disorders. Int Urogynecol J Pelvic Floor Dysfunct 2001; 12:178-86.

2. Arthanasiou S, Hill S, Gleeson C, et al. Validation of the ICS proposed pelvic organ prolapse descriptive system. Neurourol Urodyn 1995; 14:414-5.

3. Schussler B, Peschers U. Standardization of terminology of female genital prolapse according to the new ICS criteria: interexaminer reproducibility. Neurourol Urodyn 1995; 14:437-8.

4. Kobak WH, Rosenberger K, Walters MD. Interobserver variation in the assessment of pelvic organ prolapse. Int Urogynecol J Pelvic Floor Dysfunct 1996; 7:121-4.

5. Bump RC, Mattiasson A, Bo K, et al. The standardization of terminology of female pelvic organ prolapse and pelvic floor dysfunction. Am J Obstet Gynecol 1996; 175:10-17.
6. Hall AF, Theofrastous JP, Cundiff GW, et al. Interobserver and intraobserver reliability of the proposed International Continence Society, Society of Gynecologic Surgeons, and American Urogynecologic Society pelvic organ prolapse classification system. Am J Obstet Gynecol 1996; 175:1467-71.

7. International Continence Society. Committee on Standardization of Terminology. The standardization of terminology of lower urinary tract function. In: Ostergard DR, Bent AE, editors. Urogynecology and Urodynamic: theory and practice. $3^{\text {rd }}$ ed. Baltimore: Williams \& Wilkins; 1991. p.545-62.

8. Netter J, Wasferman W, Kutner MH. Applied linear statistical models: regression, analysis of variance and experimental designs. $3^{\text {rd }}$ ed. Homewood: RD Irving; 1996. p.1181.

9. Porges RF. A practical system of diagnosis and classification of pelvic relaxations. Surg Gynecol Obstet 1963; 117:769-73.

10.Baden WF, Walker TA, Lindsey JH. The vaginal profile. Tex Med 1968; 64:56-8.

11.Beecham CT. Classification of vaginal relaxation. Am J Obstet Gynecol 1980; 136:957-8.

12.Juma S, Little NA, Raz S. Evaluation of stress urinary incontinence. In: Buchsbaum HJ, Schmidt JD, editors. Gynecologic and Obstetric Urology. $3^{\text {rd }}$ ed. Philadelphia: W.B. Saunders; 1993. p.251-63.

13. Fantl JA, Newman DK, Colling J, et al. Urinary incontinence in adults: acute and chronic management. Rockville: US Department of Health and Human Services; 1996. (AHCPR Publication, $\mathrm{n}^{\circ}$ 96-0682. Clinical Practice Guideline, $\left.\mathrm{n}^{\circ} 2\right)$.

14.Black NA, Downs SH. The effectiveness of surgery for stress incontinence in women: a systematic review. Br J Urol 1996; 78:497-510.

15.Sze EH, Karram MM. Transvaginal repair of vault prolapse: a review. Obstet Gynecol 1997; 89:466-75.

16.Bland DR, Earle BB, Vitolins MZ, Burke G. Use of the pelvic organ prolapse staging system of the International Continence Society, American Urogynecologic Society, and Society of Gynecologic Surgeons in perimenopausal women. Am J Obstet Gynecol 1999; 181:1324-8.

17.Swift SE, Herring M. Comparison of pelvic organ prolapse in the dorsal lithotomy compared with standing position. Obstet Gynecol 1998; 91:961-4.

18.Barber MD, Lambers AR, Visco AG, Bump RC. Effect of patient position on clinical evaluation of pelvic organ prolapse. Obstet Gynecol 2000; 96:18-22.

19.Steele A, Mallipeddi P, Welgoss J, Soled S, Kohli N, Karram M. Teaching the pelvic organ prolapse quantitation system. Am J Obstet Gynecol 1998; 179:1458-64. 